\title{
Analysis of B7-H4 expression in metastatic pleural adenocarcinoma and therapeutic potential of its antagonists
}

\author{
Cheng Chen ${ }^{1 \dagger}$, Qiu-Xia Qu ${ }^{2 \dagger}$, Fang Xie ${ }^{3}$, Wei-Dong Zhu ${ }^{4}$, Ye-Han Zhu ${ }^{1 *}$ and Jian-An Huang ${ }^{{ }^{*}}$
}

\begin{abstract}
Background: The increasing incidence and poor outcome associated with malignant pleural effusion (MPE) requires finding an effective treatment for this disease. Inhibitory B7-H4 is expressed in many different human cancers but its role in malignant pleural tissue has yet to be established.

Methods: Here, patients with metastatic pleural adenocarcinoma (MPA) or with early-stage lung adenocarcinoma were clinically and statistically analyzed. Immunohistochemistry and confocal microscopy were used to determinate the expression of B7-H4 in the cancer cells. By using MPE model, we sought to a potential immunotherapy for MPE with anti-B7-H4 mAb.

Results: When compared to early-stage lung adenocarcinoma, MPA possessed higher level of nuclei membranous B7-H4 and lower cytoplasmic B7-H4 expression. Also, nuclei membranous B7-H4 expression was found to be positively correlated to Ki-67 expression, and indicated a possible poor prognosis of MPA. In mouse MPE model, intra-pleurally injection of anti-B7-H4 mAb effectively suppressed MPE formation.
\end{abstract}

Conclusions: Taken together, our data was in support of the significance of B7-H4 expression in MPA, which also suggest it warrants further exploration for potential immunotherapy of MPE.

Keywords: Metastatic pleural neoplasms, Adenocarcinoma, B7-H4, Immunotherapy

\section{Background}

Clinically, pleural effusion (PE) is a common condition caused by malignant tumors, as well as benign diseases [1]. For malignant pleural effusion (MPE), malignant cells would establish the way to the pleura through direct extension or through lymphatic or hematogenous spread $[2,3]$. The reason for the aggressive behavior of malignant cells can be attributable to their molecular aberrations. So, the better knowledge of histopathological features and the available molecules could clinically been applied for diagnosis and therapy of MPE.

B7-H4, an cell surface immunomodulatory glycoprotein, was primarily found to express on antigen-presenting cells and in some non-hematopoietic tissues. It has also been

\footnotetext{
*Correspondence: zhuyehansz@sina.com; huang_jian_an@163.com ${ }^{\dagger}$ Equal contributors

${ }^{1}$ Respiratory Department, The First Affiliated Hospital of Soochow University, 188 Shizi Street, Suzhou 215006, China

Full list of author information is available at the end of the article
}

demonstrated that B7-H4 is broadly expressed in many malignant tumors including carcinomas of the hepatocellular, lung, osteosarcoma and ovarian, and it contributes to the tumor immune escape [4-7]. It has also been reported that measurement of sB7-H4 might bel diagnostic value for MPE [8]. However, the B7-H4 expression in metastatic pleural adenocarcinoma (MPA) has not been widely reported. Furthermore, since PD-L1 and PD-1 $\mathrm{mAb}$ revolutionized cancer immunotherapy, an impressive variety of clinic trials of checkpoint blockade were already underway or planned [9]. At this stage, as another checkpoint, B7-H4 might also participate to tumor progression and be a candidate target to cancer immunotherapy $[10,11]$.

Also, the detection of B7-H4 in cytoplasm of tumor cells suggested it might have some unanticipated function that is different from membrane B7-H4. For example, Zhang $\mathrm{L}$ et al. revealed that $\mathrm{B} 7-\mathrm{H} 4$ promote renal cell carcinoma progression and cell proliferation 
through translocating into nucleus [12]. It was also suggested that the intracellular $\mathrm{B} 7-\mathrm{H} 4$ appears to prevent Fas/FasL-mediated bile duct epithelial cells apoptosis during the progression of primary biliary cirrhosis (PBC) [13].

In present study, we compared the expressing profile of B7-H4 in MPA to that in early-stage lung adenocarcinoma, and to determine whether B7-H4 could be used as a carcinogenic factor for MPE. Finally, by using MPE model, we sought to further investigate whether anti-B7- $\mathrm{H} 4 \mathrm{mAb}$ treatment could be used as a potential immunotherapy for MPE.

\section{Methods}

\section{Cell line and $\mathrm{mAb}$}

Six to eight week-old female C57BL/6 mice $\left(\mathrm{H}-2^{\mathrm{b}}\right)$ were purchased from Chinese Academy of Sciences, Shanghai Institutes for Biological Sciences, Experimental Animal Center. Lewis lung carcinoma cell line (LLC) was purchased from Chinese Academy of Sciences, Shanghai Institutes for Biological Sciences. The cell was cultured in RPMI1640 supplemented with 10\% fetal calf serum (FCS), $2 \mathrm{mM}$ L-glutamine, $100 \mathrm{mg} / \mathrm{mL}$ streptomycin, $100 \mathrm{U} / \mathrm{mL}$ penicillin, and $50 \mathrm{mM} \mathrm{2-ME}$. The cell was incubated at $37{ }^{\circ} \mathrm{C}$ with $5 \% \mathrm{CO}_{2}$.

\section{Patients}

Twenty-three patients with PE were admitted at The First Affiliated Hospital of Soochow University from 2011 to 2016. All patients were proven histologically diagnosis of MPA by pleural biopsy under thoracoscope. The subjects also comprised 9 patients with solitary pulmonary nodules (SPN) who underwent thoracotomy or selected videoassisted thoracic surgery (VATS) between 2012 and 2015. Clinicopathologic information included complete history, age, sex and histology subtype.

\section{Immunohistochemistry}

Formalin-fixed, paraffin-embedded samples were cut ( $4 \mu \mathrm{m}$-thick sections) and placed on silane-covered slides. Morphological assessment was obtained by hematoxylineosinsaffron staining. In brief, after dewaxing, inactivating endogenous peroxidase activity and blocking crossreactivity with $3 \% \mathrm{BSA}$, all sections were incubated at $37{ }^{\circ} \mathrm{C}$ for $1 \mathrm{~h}$ with diluted solution of the $\mathrm{B} 7-\mathrm{H} 4 \mathrm{mAb}$ (ployclonal antibody, Novus) and Ki-67 mAb (Genetech, clone GM001), respectively. Location of the primary antibodies was achieved by subsequent reaction with a horseradish peroxidase-conjugated anti-primary antibody. Negative controls were established with mouse IgG (BD PharMingen) by replacing the primary antibody.

\section{Evaluation of immunohistochemical results}

Five high-power fields were randomly selected. The score of the B7-H4 staining was categorized into five semiquantitative classes based on the percentages of positive tumor cells: 0 (<5\%), 1 (6-25\%), 2 (26-50\%), 3 (51-75\%) and $4(>75 \%)$. The intensity of cellular staining was also assessed semi-quantitatively on a scale of $0-3$ as follows: 0 (negative), 1 (weakly positive), 2 (moderately positive) and 3 (strongly positive). The scores of intensity and the percentage gave rise to the final staining result: - $(0),+$ $(1-2),++(3-4)$, and $+++(5-7)$. During statistical analysis, tumors have a final result of - or + , which showed a no/ low immunoreactivity, compared to tumors with result of ++ or +++ as the high immunoreactivity. The expression of Ki-67 was evaluated according to the percent of its staining and scored.

\section{Confocal microscopy}

MPA sections were blocked with 3\% BSA for $30 \mathrm{~min}$ and incubated for $1 \mathrm{~h}$ with PE-labeled anti-B7-H4 Ab. After washes, sections were incubated with 4,6-diamidino-2phenylindole (DAPI, Roche Diagnostics). Fluorescence was visualized with Axiophot 1. Images were captured with an Axiocam color charge-coupled device camera and analyzed with AxioVision software (Carl Zeiss) [14].

\section{MPE model}

Murine MPE models were prepared according to the methods described by Servais EL [15] by intrapleural injection of LLC cells $\left(10^{6} /\right.$ mouse). Briefly, slowly insert the needle to the intercostal space between the diaphragm and lung at a shallow angle of $15^{\circ}$, needle bevel up, entering the pleural space through the diaphragm. At days 14

Table 1 B7-H4 expression in metastatic pleural adenocarcinoma

\begin{tabular}{|c|c|c|c|c|}
\hline \multirow[t]{2}{*}{ Parameter } & \multicolumn{2}{|c|}{ Nuclear membrane } & \multicolumn{2}{|c|}{ Cytoplasm } \\
\hline & Low & High & Low & High \\
\hline \multicolumn{5}{|c|}{ Age (MPN, median = 58) } \\
\hline$>58$ & 1 & 10 & 6 & 5 \\
\hline$\leq 58$ & 4 & 8 & 6 & 6 \\
\hline \multicolumn{5}{|c|}{ Age (LAC-stage I, median = 62) } \\
\hline$>62$ & 4 & 0 & 0 & 4 \\
\hline$\leq 62$ & 4 & 1 & 0 & 5 \\
\hline \multicolumn{5}{|l|}{ MPA } \\
\hline Adenocarcinoma & 5 & $18^{*}$ & 12 & $11^{* *}$ \\
\hline LAC-Stage I & 8 & 1 & 0 & 9 \\
\hline $\mathrm{AAH}$ & 2 & 0 & 0 & 2 \\
\hline AIS & 2 & 1 & 0 & 3 \\
\hline Lepidic & 4 & 0 & 0 & 4 \\
\hline
\end{tabular}

${ }^{*} P<0.01$ compared to the LAC-Stage I, ${ }^{* *} P=0.012$ compared to the LAC-Stage I. Metastatic pleural adenocarcinoma, MPA, LAC-stage I, lung adenocarcinoma-stage I 


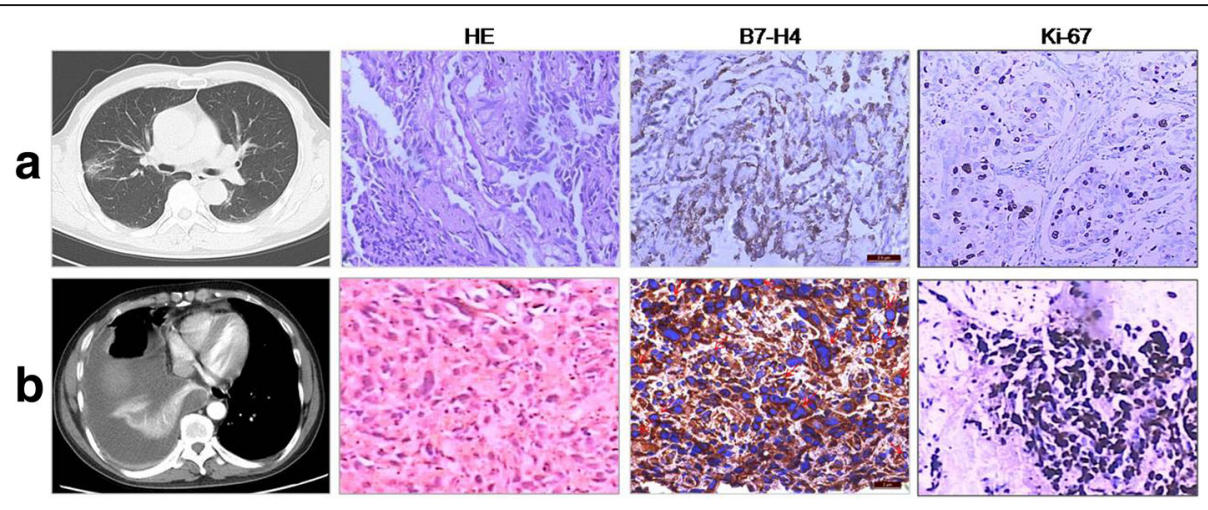

Fig. 1 Immunostaining of B7-H4 and Ki-67 in lung adenocarcinoma. a line, CT scan shows opacity with ground-glass in the right lung, HE staining confirmed lepidic predominant adenocarcinoma with high differentiation, IHC demonstrated a negative nuclei membranous B7-H4 and low Ki-67 staning. b line, CT scan shows pleural effusion in the right lung, HE staining confirmed MPA, IHC demonstrated a high nuclei membranous B7-H4 and strong Ki-67 staning, (red arrow, ×40). One representative data was showed

after intrapleural injection of LLC cells, all mice were performed CT scan to confirm the formation of pleural effusion. The CT images were captured with the mouse under anesthesia in a common animal holder.

\section{In vivo experiment}

Mice with pleural effusion were received local injection of $50 \mu \mathrm{g}$ anti-B7-H4 neutralized mAb (clone 9, eBioscience), or $50 \mu \mathrm{g}$ mouse isotype $\mathrm{mAb}$ (eBioscience), respectively. After 7 days, CT scan was repeated.

\section{Statistical analysis}

Statistical analysis was performed with SPSS statistical software (Version 19.0; SPSS Inc., Chicago, IL, USA). Overall survival (OS) was calculated from diagnosis of disease until the last follow-up for alive patients or until death due to any cause. OS analysis was carried out using the Kaplan-Meier curves [16]. The difference of B7-H4 expression in MPA and early-stage lung cancer was evaluated by using $\mathrm{x}^{2}$ tests. $P$ values $<0.05$ were considered significant.

\section{Results}

\section{Patient population}

Baseline characteristics of all subjects were listed in Table 1. 23 patients underwent the pleural biopsy under endoscope and diagnosed by MPA. 9 patients underwent the surgery and diagnosed by stage I, which included 2 atypical adenomatous hyperplasia (AAH), 3 adenocarcinoma in situ (AIS), 4 lepidic adenocarcinoma (LA).

\section{B7-H4 expression in MPA}

B7-H4 was found to express along the nuclear membrane in 18 (78.3\%) of 23 MPA by immunohistochemistry analysis. High cytoplasmic immunostaining of B7-H4 was found to be in $47.8 \%(11 / 23)$ cases (Table 1 and Figs. 1, 2, 3). Additionally, cytoplasmic B7-H4 and nuclei membranous B7-H4 immunostaining were also confirmed in situ by Confocal Microscopy (Fig. 2). Then, we used patients with AAH, AIS or LA as early-stage of lung cancer. Of note, when compared to MPA, earlystage of lung cancer possessed higher level of cytoplasmic B7-H4, and only rare cases $(11.1 \%)$ were stained positively with nuclei membranous B7-H4 (Table 1 and Figs. 1, 3). Taken together, our data demonstrate a distinct B7-H4 expression between early-stage of lung adenocarcinoma and MPA, decrease of cytoplasmic and occurrence of nuclear membranous $\mathrm{B} 7-\mathrm{H} 4$ was associated with the increase of malignancy of cancer cells and development of MPA.

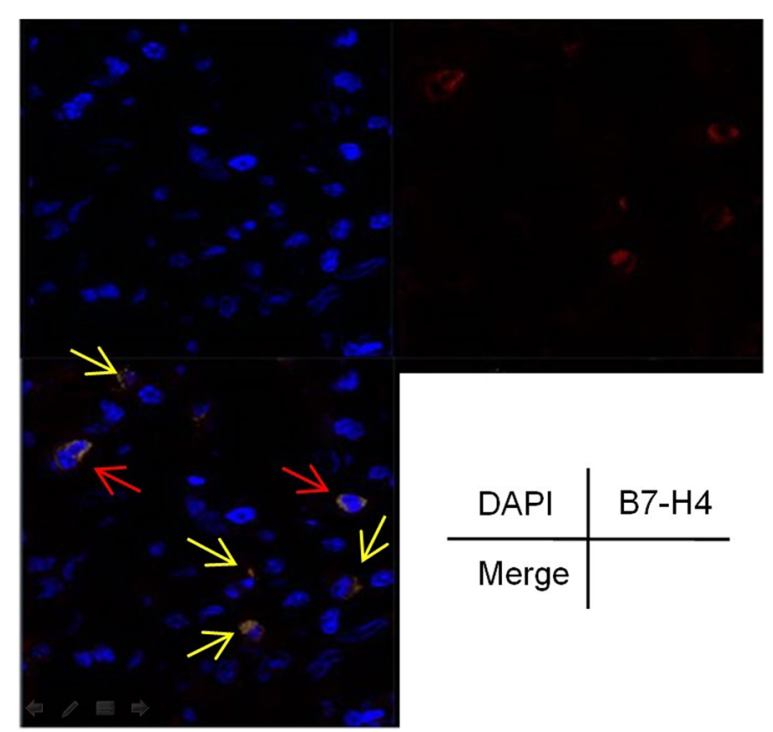

Fig. 2 The expression of B7-H4 in the MPA was investigated by Confocal Microscopy. Images were captured with an Axiocam color charge-coupled device camera, one representative nuclei membranous B7-H4 (red arrow) and cytoplasmic B7-H4 (yellow arrow) was shown 


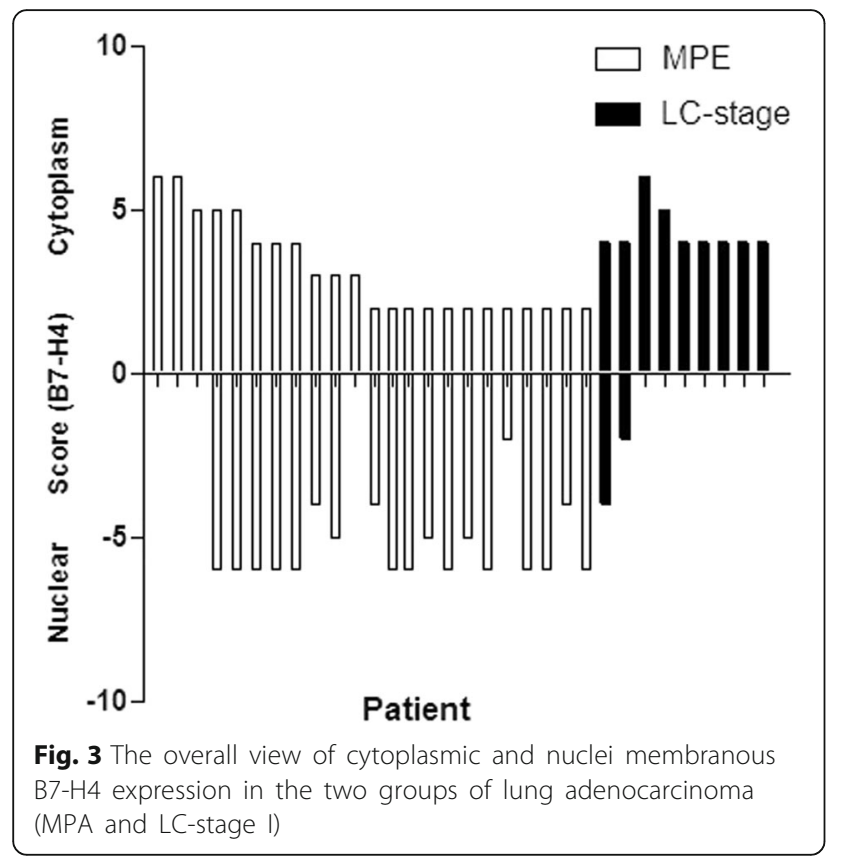

\section{Expression of B7-H4 and $\mathrm{Ki}-67$ in MPA}

Furthermore, we also assessed expression of Ki-67, an identified proliferation antigen of the carcinomas, to explore whether B7-H4 expression is associated with increased cancer cell proliferation. As shown in Fig. 4, Ki-67 immunostaining was correlated to nuclei membranous B7-H4 $(P<0.05)$, but not to its expression in cytoplasm $(P>0.05)$, which suggested that nuclei membranous $\mathrm{B} 7-\mathrm{H} 4$ may be regarded as a proliferative factor for MPA.

\section{Impact of B7-H4 expression on survival of MPA}

Additionally, we analyzed the outcomes for patient's overall survival according to $\mathrm{B} 7-\mathrm{H} 4$ staining patterns. For B7-H4 expression, patients were grouped as "high" or "low" using the nuclei membranous immunostaining. 23 cases had follow-ups for 24 months for observing OS (Fig. 4c). Median survival for high nuclei membranous
B7-H4 patients was 10 months and 15 months for B7$\mathrm{H} 4$ low patients, indicating that nuclei membranous B7-H4 expression has possible impacts on survival of MPA patients. Due to low patient' number here, it would attach statistical significance if we expanded the sample size.

\section{Efficacy of B7-H4 mAb on malignant pleural effusion}

To determine whether $\mathrm{B} 7-\mathrm{H} 4$ expression affects formation of MPE, we tested the volume of MPE by CT scan in MPE mice before and after anti-B7-H4 mAb treatment. At the same slice, we can semi-quantitative the MPE by fluid sonolucent area noticed in mediastinal window. We showed that the intra-pleurally anti-B7-H4 mAb treatment significantly suppressed MPE (Fig. 5).

\section{Discussion}

B7-H4 has been found to be expressed at both mRNA and protein levels in many types of cancers, and the negative clinical associations of B7-H4 was found in lung, kidney, prostate, and gastrointestinal cancers. B7-H4 deficiency in mice resulted in significant protection from lung metastases and increased survival in the 4T1 tumor model. Mechanically, B7-H4 prompted metastasizing cancer cells to escape local antitumor immune responses through interactions with the innate and adaptive immune cell [17]. However, some studies also showed a role for B7-H4 in enhancing antitumor immunity. Clinically, breast cancer patients with increased $\mathrm{B} 7-\mathrm{H} 4$ expression showed a prolonged time to recurrence [18]. It was also demonstrated an effective immune regulation due to the B7-H3, but not the B7-H4 in the cancer microenvironment [19]. Given this unexpected phenotype, we extended our analysis of B7-H4 in MPA.

Here, $78.3 \%$ and $47.8 \%$ of MPA tissues were found to express nuclear membranous and cytoplasmic B7-H4 respectively. When using early-stage lung cancer as control, it is indicated that there was a distinct B7-H4 expression possessed by MPA. In brief, it was noted that the almost all AAH, AIS and LA were absent in expression of B7-H4
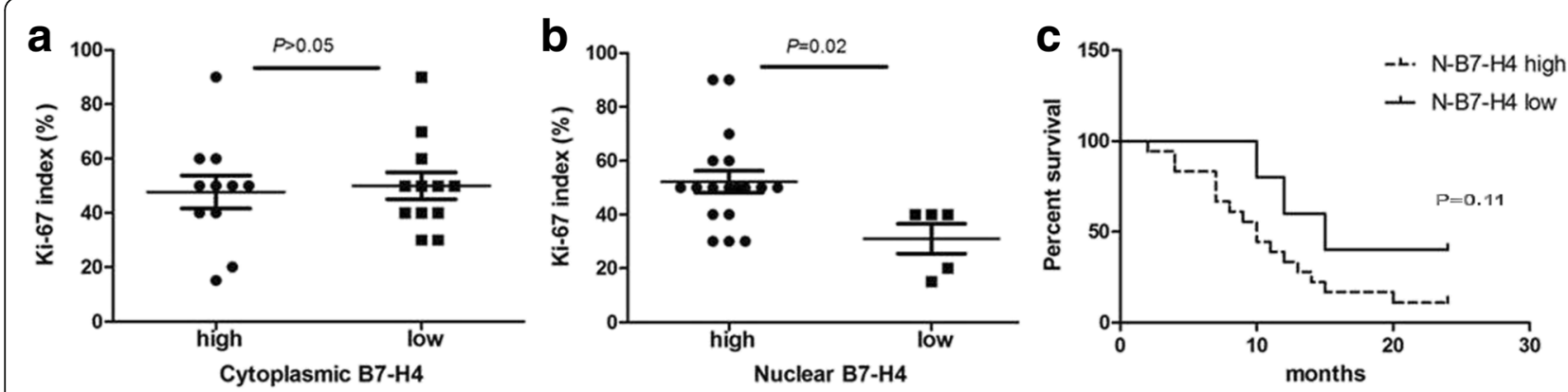

Fig. 4 Correlation of the Ki-67 index with cytoplasmic (a) and nuclei membranous (b) B7-H4 in MPA patients was shown respectively. Kaplan-Meier survival curves for MPA patients according to expression level of nuclei membranous B7-H4 was shown in (c) 


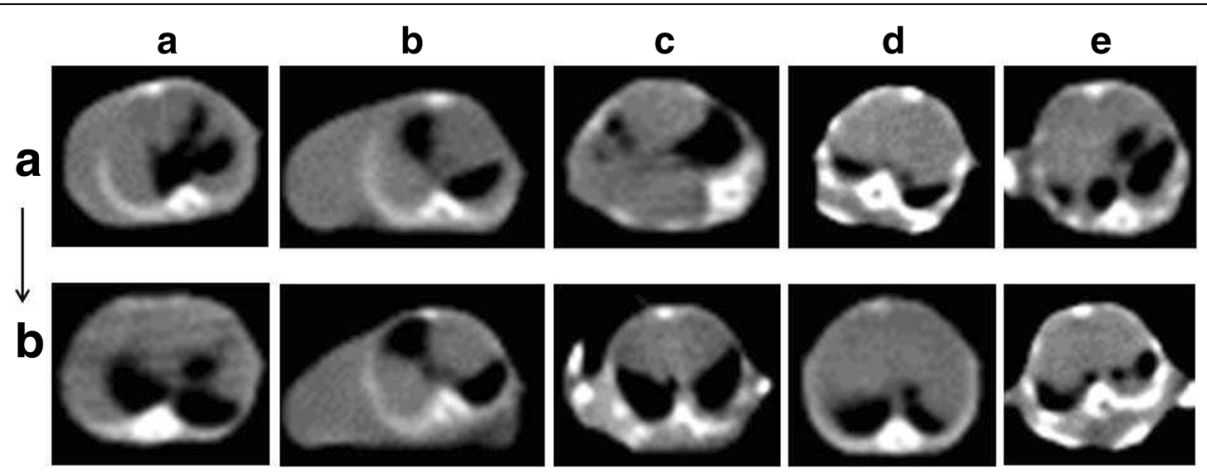

Fig. 5 The overall view of MPE in the five mice was shown. $\mathbf{a}$, before anti-B7-H4 mAb injection, $\mathbf{b}$, after anti-B7-H4 mAb injection

by nuclear membrane, instead of high cytoplasmic staining. In according to our data, we hypothesized that B7-H4 might work as a nuclear shuttling protein along with tumor progress, which mean poorer differentiated and more invasive adenocarcinoma would have higher nuclear membranous B7-H4 expression.

As previously reported, in immune-deficient mice, over-expression of $\mathrm{B} 7-\mathrm{H} 4$ could promote tumorigenesis of ovarian cancer by increased proliferation, adhesion, migration and invasion. In others' study, over-expression of B7-H4 on epithelial cells could result in malignant cellular transformation, perhaps though protecting the pre-transformed cells from apoptosis. So, it implied that B7-H4 might have a direct effect on tumorigenesis independent of immune property $[12,13,20]$. As supported, our data indicated that nuclei membranous B7-H4 has a positive correlation to the expression of $\mathrm{Ki}-67$, and it might have a functional activity in promoting cancer cell growth.

In the last decade, immune checkpoint inhibition had led to major therapeutic advances in tumor oncology. Given that B7-H4 is widely expressed in all examined cancer specimens and its inhibitory immune function, this paradigm called for the development of novel cancer immunotherapy strategies by targeting B7-H4. Taking advantage of an orthotopic model that faithfully mimics human pleural malignancy, we evaluated administration of B7-H4 mAb treatment to MPE. As expected, it was found that intra-pleurally administered B7-H4 mAb could decrease MPE production. It was supported by Adusumilli's study, which demonstrated that intra-pleurally administered chimeric antigen receptor (CAR)-engineered $\mathrm{T}$ cells induce long-term complete remissions in malignant pleural mesothelioma (MPM) model [21]. Also, immune checkpoint inhibitors have been linked to the development of certain adverse events, such as cutaneous, hepatic and gastrointestinal toxicities, which commonly described as immune-related adverse events (irAEs) [22-24]. We believed that the routine of intra-pleurally administered $\mathrm{B} 7-\mathrm{H} 4 \mathrm{mAb}$ will be utilized in clinical studies, especially can decrease the risk of irAEs induced by systemic administration.

\section{Conclusions}

Taken together, in the present study, our data in support of the functional role of B7-H4 in MPA come from evidence of $\mathrm{B} 7-\mathrm{H} 4$ expression on cancer cells and therapeutic efficacy of B7-H4 mAb on MPE. We believe that $\mathrm{B} 7-\mathrm{H} 4$ could become a valuable tool to add to the oncologist's toolbox for predicting the prognosis of MPA patients. Although patient' number was relatively low here, it has deepen our knowledge about biomarkers for analysis of the occurrence of MPA. This may be possible when B7-H4 was investigated on a larger number of cases in the future. Consequently, B7-H4 blockade could also offer a new therapeutic strategy for MPE [25].

\section{Abbreviations}

AAH: atypical adenomatous hyperplasia; AIS: adenocarcinoma in situ; B7-H4: B7 homlogs 4; CAR: chimeric antigen receptor; DAPI: 4,6-diamidino-2phenylindole; IrAEs: immune-related adverse events; LA: lepidic adenocarcinoma; Mc Ab: monoclonal antibody; MPM: malignant pleural mesothelioma; PE: pleural effusion

\section{Acknowledgements}

The authors thank Prof. Jim Xiang (Cancer Research Unit, Saskatchewan Cancer Agency, University of Saskatchewan, Saskatoon, Canada) for providing constructive criticism and helpful suggestions for this manuscript.

\section{Author contributions}

Conception \& design - CC, YHZ, and JAH. Patient selection and patient data collection - CC. Immunostaining evaluation - QXQ, FX, and WDZ. Animal model - QXQ. Statistical analyses - CC. Manuscript drafting and revision - CC. All authors have read and approved the manuscript.

\section{Funding}

This work was supported by the Project of National Natural Science Foundation of China (81672280), Societal and Developmental Project of Suzhou City (SYS201630), Key laboratory of Suzhou City (SZS201617), Clinical Medical Center of Suzhou (SZZX201502), Jiangsu Province's Key Discipline of Medicine (ZDXKB2016007) and Medical Youth of Jiangsu Province (QNRC2016748). The funders had no role in the study design, data collection and analysis, decision to publish, or preparation of the manuscript. 


\section{Availability of data and materials}

The datasets supporting the conclusions of this article are included within the article. More datasets of the current study are available from the corresponding author on reasonable request.

\section{Ethics approval and consent to participate}

The study was approved by the committee for medical and health research ethics of the First Affiliated Hospital of Soochow University. Medical personnel explained to each patient the aim of the pathologic analysis. All cases signed a written consent. All methods and animal experimental protocols were approved and performed in accordance with the relevant guidelines and regulations of committee for medical and health research ethics of the First Affiliated Hospital of Soochow University.

\section{Consent for publication}

Not applicable.

\section{Competing interests}

The authors declare that they have no competing interests.

\section{Publisher's Note}

Springer Nature remains neutral with regard to jurisdictional claims in published maps and institutional affiliations.

\section{Author details}

'Respiratory Department, The First Affiliated Hospital of Soochow University, 188 Shizi Street, Suzhou 215006, China. ${ }^{2}$ Clinical Immunology Laboratory, The First Affiliated Hospital of Soochow University, 788 Renmin Road, Suzhou 215007, China. ${ }^{3}$ Pathology Division, Soochow University, 1 Shizi Street, Suzhou 215006, China. ${ }^{4}$ Pathology Department, The First Affiliated Hospital of Soochow University, 188 Shizi Street, Suzhou 215006, China.

Received: 21 April 2017 Accepted: 28 August 2017

Published online: 18 September 2017

\section{References}

1. Light RW. Clinical practice: pleural effusion. N Engl J Med. 2002;346(25): 1971-7.

2. Ye ZJ, Zhou Q, YY G, Qin SM, Ma WL, Xin JB, Tao XN, Shi HZ. Generation and differentiation of $\mathrm{LL}-17$-producing $\mathrm{CD} 4+\mathrm{T}$ cells in malignant pleural effusion. J Immunol. 2010;185(10):6348-54.

3. Chen $Y Q$, Shi $H Z$, Qin $X J$, Mo WN, Liang $X D$, Huang $Z X$, Yang HB, Wu C. $\mathrm{CD} 4+C D 25+$ regulatory $T$ lymphocytes in malignant pleural effusion. Am 」 Respir Crit Care Med. 2005;172(11):1434-9.

4. Hong B, Qian Y, Zhang H, Sang YW, Cheng LF, Wang Q, Gao S, Zheng $M$, Yao HP. Expression of B7-H4 and hepatitis B virus $X$ in hepatitis $B$ virus-related hepatocellular carcinoma. World J Gastroenterol. 2016;22(18):4538-46.

5. Schalper KA, Carvajal-Hausdorf D, McLaughlin J, Altan M, Velcheti V, Gaule P, Sanmamed MF, Chen L, Herbst RS, Rimm DL. Differential expression and significance of PD-L1, IDO-1 and B7-H4 in human lung cancer. Clin cancer res. Clin Cancer Res. 2017;23(2):370-8.

6. Dong $\mathrm{Q}, \mathrm{Ma}$ X. B7-H4 expression is associated with tumor progression and prognosis in patients with osteosarcoma. Biomed Res Int. 2015:156432.

7. Salceda S, Tang T, Kmet M, Munteanu A, Ghosh M, Macina R, Liu W, Pilkington G, Papkoff J. The immunomodulatory protein B7-H4 is overexpressed in breast and ovarian cancers and promotes epithelial cell transformation. Exp Cell Res. 2005:306(1):128-41.

8. Xu C, Qian L, Yu L, Zhang X, Wang Q. Evaluation of serum and pleural levels of soluble $\mathrm{B} 7-\mathrm{H} 4$ in lung cancer patients with pleural effusion. Biomarkers. 2015;20(4):271-4.

9. Meng $X$, Huang Z, Teng F, Xing L, Predictive YJ. Biomarkers in PD-1/PD-L1 checkpoint blockade immunotherapy. Cancer Treat Rev. 2015:41(10):868-76.

10. Dangaj D, Lanitis E, Zhao A, Joshi S, Cheng Y, Sandaltzopoulos R, Ra HJ, Danet-Desnoyers G, Powell DJ Jr, Scholler N. Novel recombinant human b7-h4 antibodies overcome tumoral immune escape to potentiate T-cell antitumor responses. Cancer Res. 2013;73(15):4820-9.

11. Leong SR, Liang WC, Wu Y, Crocker L, Cheng E, Sampath D, Ohri R, Raab H, Hass PE, Pham T, et al. An anti-B7-H4 antibody drug conjugate for the treatment of breast cancer. Mol Pharm. 2015;12(6):1717-29.
12. Zhang L, Wu H, Lu D, Li G, Sun C, Song H, Li J, Zhai T, Huang L, Hou C, et al. The costimulatory molecule B7-H4 promote tumor progression and cell proliferation through translocating into nucleus. Oncogene. 2013;32(46): $5347-58$.

13. Chen Y, Guo G, Guo S, Shimoda S, Shroyer KR, Tang Y, Intracellular WY. B7$\mathrm{H} 4$ suppresses bile duct epithelial cell apoptosis in human primary biliary cirrhosis. Inflammation. 2011;34(6):688-97.

14. Chen C, Zhu YB, Shen Y, Zhu YH, Zhang XG, Huang JA. Increase of circulating B7-H4-expressing CD68+ macrophage correlated with clinical stage of lung carcinomas. J Immunother. 2012;35(4):354-8.

15. Servais EL, Colovos C, Kachala SS, Adusumilli PS. Pre-clinical mouse models of primary and metastatic pleural cancers of the lung and breast and the use of bioluminescent imaging to monitor pleural tumor burden. Curr Protoc Pharmacol. 2011; Chapter 14:Unit14.21

16. Cedrés S, Ponce-Aix S, Pardo-Aranda N, Navarro-Mendivil A, Martinez-Marti A, Zugazagoitia J, Sansano I, Montoro MA, Enguita A, Felip E. Analysis of expression of PTEN/PI3K pathway and programmed cell death ligand 1 (PD-L1) in malignant pleural mesothelioma (MPM). Lung Cancer. 2016;96:1-6.

17. Abadi YM, Jeon H, Ohaegbulam KC, Scandiuzzi L, Ghosh K, Hofmeyer KA, Lee JS, Ray A, Gravekamp C, Zang X. Host B7x promotes pulmonary metastasis of breast cancer. J Immunol. 2013;190(7):3806-14.

18. Rahbar R, Lin A, Ghazarian M, Yau HL, Paramathas S, Lang PA, Schildknecht A, Elford AR, Garcia-Batres C, Martin B, et al. B7-H4 expression by nonhematopoietic cells in the tumor microenvironment promotes antitumor immunity. Cancer Immunol Res. 2015;3(2):184-95.

19. Kreymborg K, Haak S, Murali R, Wei J, Waitz R, Gasteiger G, Savage PA, van den Brink MR, Allison JP. Ablation of B7-H3 but not B7-H4 results in highly increased tumor burden in a murine model of spontaneous prostate cancer. Cancer Immunol Res. 2015;3(8):849-54.

20. Cheng L, Jiang J, Gao R, Wei S, Nan F, Li S, Kong B. B7-H4 expression promotes tumorigenesis in ovarian cancer. Int J Gynecol Cancer. 2009;19(9):1481-6.

21. Adusumilli PS, Cherkassky L, Villena-Vargas J, Colovos C, Servais E, Plotkin J, Jones DR, Sadelain M. Regional delivery of mesothelin-targeted CAR T cell therapy generates potent and long-lasting CD4-dependent tumor immunity. Sci Transl Med. 2014;6(261):261ra151.

22. Gangadhar T, Vonderheide R. Mitigating the toxic effects of anticancer immunotherapy. Nat Rev Clin Oncol. 2014;11(2):91-9.

23. Weber J, Kahler K, Hauschild A. Management of immune-related adverse events and kinetics of response with ipilimumab. J Clin Oncol. 2012;30(21):2691-7.

24. Abdel-Rahman O, ElHalawani H, Fouad M. Risk of elevated transaminases in cancer patients treated with immune checkpoint inhibitors: a meta-analysis. Expert Opin Drug Saf. 2015;14(10):1507-18.

25. Smith JB, Lanitis E, Dangaj D, Buza E, Poussin M, Stashwick C, Scholler N, Powell DJ Jr. Tumor regression and delayed onset toxicity following B7-H4 CAR T cell therapy. Mol Ther. 2016;24(11):1987-99.

\section{Submit your next manuscript to BioMed Central and we will help you at every step:}

- We accept pre-submission inquiries

- Our selector tool helps you to find the most relevant journal

- We provide round the clock customer support

- Convenient online submission

- Thorough peer review

- Inclusion in PubMed and all major indexing services

- Maximum visibility for your research

Submit your manuscript at www.biomedcentral.com/submit
Ciomed Central 